\title{
Carnets
}

Revue électronique d'études françaises de l'APEF

Deuxième série - 12 | 2018

Théorie Mimétique et Études Littéraires

\section{René Girard, Don Quichotte et la comédie}

Le conflit et l'appropriation mimétique dans le fonctionnement du comique

\section{Toufic El-Khoury}

\section{CpenEdition}

1 Journals

Édition électronique

URL : https://journals.openedition.org/carnets/2427

DOI : $10.4000 /$ carnets. 2427

ISSN : 1646-7698

Éditeur

APEF

\section{Référence électronique}

Toufic El-Khoury, «René Girard, Don Quichotte et la comédie », Carnets [En ligne], Deuxième série - 12 |

2018, mis en ligne le 19 janvier 2018, consulté le 22 septembre 2021. URL : http://

journals.openedition.org/carnets/2427 ; DOI : https://doi.org/10.4000/carnets.2427

Ce document a été généré automatiquement le 22 septembre 2021.

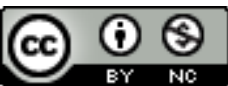

Carnets est mis à disposition selon les termes de la licence Creative Commons - Atribution - Pas d'utilisation commerciale 4.0 International. 


\title{
René Girard, Don Quichotte et la comédie
}

Le conflit et l'appropriation mimétique dans le fonctionnement du comique

\author{
Toufic El-Khoury
}

\section{René Girard et la médiation externe du désir}

1 René Girard n'est pas particulièrement connu pour son travail sur la comédie. Ses premiers textes traitant directement d'œuvres comiques (littéraires et théâtrales) se sont intéressés aux comédies de Shakespeare et à Don Quichotte de Cervantès, parodie des romans de chevalerie très populaires aux $\mathrm{XVI}^{\mathrm{e}}$ et $\mathrm{XVII}^{\mathrm{e}}$ siècles. Vingt ans plus tard, dans un texte rédigé en 1987 et repris dans La voix méconnue du réel en 2002, il traite directement du comique et de ses mécanismes, bien qu'il aborde la question sous l'angle de ses propres thèses. Mais si son apport à l'étude du comique demeure périphérique, voire anecdotique, dans l'ensemble de son œuvre, il se révèle néanmoins, par certains aspects, potentiellement intrigant.

2 Toute tentative de Girard concernant, de près ou de loin, des œuvres comiques intervient dans le cadre d'une instrumentalisation potentielle de ces œuvres dans le cadre de la mise en place de sa théorie mimétique. Le cas de Don Quichotte n'échappe pas à la règle et est dans ce sens révélateur.

En lisant le roman de Cervantès, Girard s'intéresse principalement à l'incapacité du protagoniste à aligner ses désirs et ses actions sur ceux de ses modèles fictifs, les héros des romans de chevalerie dont il lit, jusqu'à la folie, les exploits. La prémisse de l'œuvre sert de point de départ, et de principale motivation, pour étoffer sa thèse du mimétisme en tant que moteur central, rarement avoué, du désir humain. Plus précisément, dans le cas du personnage de Don Quichotte, il identifie une forme particulière du désir mimétique: la médiation externe du désir qui met en lumière la disposition du personnage à imiter et à se conformer aux actes d'un modèle qui n'appartient pas à son propre univers diégétique. Contrairement à la première forme définie par Girard, la 
médiation interne du désir, qui a pour conséquence une compétition violente entre le sujet et le médiateur, le rapport du sujet au modèle dans la médiation externe ne génère pas de rivalité directe entre eux. Le conflit est plutôt intériorisé, « le désir selon l'Autre ", comme il l'affirme dès Mensonge romantique et Vérité romanesque, "[étant] toujours le désir d'être un Autre » (Girard, 1999 : 101).

Cette médiation, qui suggère l'incapacité de désirer sans se référer continuellement à des modèles intouchables - puisque n'appartenant pas à la même sphère d'action du sujet -, est l'un des fers de lance du projet anthropologique à venir de Girard. Mais elle n'est pas propre à l'univers comique. Elle illustre surtout un rapport ambivalent à la littérature - et à tout art narratif -, Don Quichotte n'étant qu'une projection géniale de notre propre " asservissement » à des codes de conduite et de vie sublimés.

Cette thèse s'est révélée passionnante pour les tenants de disciplines des sciences humaines. C'est dans la psychologie et la sociologie que les premières réactions aux intuitions de Girard ont eu lieu. Francis Farrugia, sociologue français, introduit le concept de "syndrome narratif ", analogue à celui de médiation externe de Girard, en prenant également pour modèles littéraires Don Quichotte et Emma Bovary ${ }^{1}$.Il fait état d'un double niveau d'imitation: le premier identifie la narration comme syndrome, définie comme la disposition du héros - comique dans le cas du personnage de Cervantès - à traduire le système de valeurs chevaleresques à son propre univers, en y appliquant toutes ses actions ultérieures, avec les résultats que l'on connait: «[le personnage] vit comme il est écrit ».

6 À plusieurs reprises, Girard affirme lui-même que cette 'vérité' mimétique, qu'il a pu identifier dans les textes littéraires, il ne fait que la répéter, certes de manière systématique, après qu'elle ait été déjà suggérée par bien d'autres - bien que selon lui les philosophes, en ce qui concerne la nature du désir, aient été finalement moins perspicaces que les romanciers, les poètes et les dramaturges.

7 Dans le cadre de la médiation externe du désir, c'est le roman, ou tout art narratif en général, qui influe sur les « conduites individuelles et collectives » d'une communauté le lectorat d'une œuvre littéraire, l'audience d'une pièce de théâtre ou d'un film. La réalité imite donc la fiction. Girard souligne la même relation causale entre littérature et vie, et la place qu'occupent les récits dans la construction de nos modèles de désir, précisant que «la littérature et la vie se confondent, non pas parce que la littérature copie la vie, mais parce que la vie copie la littérature. L'harmonie se fait au niveau d'une imposture générale » (Girard, $2000: 143$ ).

8 Girard remonte jusqu'à l'expérience de Dante, décrite dans La Divine comédie, pour retrouver de premières traces de cette contamination de notre comportement amoureux par les récits, mythiques et littéraires. Dans le chant V de l'Enfer, Dante rencontre Francesca da Rimini, amante de son beau-frère Paolo Malatesta, et qui fut tuée, avec son amant, par son mari. L'histoire de ce couple adultère est devenue, grâce au poète florentin, un des mythes d'amour les plus populaires de la littérature romantique, dès la fin du XviII ${ }^{e}$ siècle. Mais pour Girard, l'œuvre de Dante est soumise sous l'impulsion du mouvement romantique à une interprétation critique qui en dénature le propos. Paolo et Francesca deviennent ainsi de nouveaux symboles, au même titre que Tristan et Yseut, de l'amour-passion: "Dans l'esprit d'innombrables lecteurs romantiques et modernes le décor infernal, si remarquable soit-il sur le plan esthétique, n'est qu'un hommage un peu vide aux conventions morales et théologiques de l'époque » (Girard, $2000:$ 197). 
Il suffirait dans ce sens de relire Dante pour deviner dans l'histoire de Paolo et Francesca une relation mimétique externe, où des modèles littéraires jouent un rôle crucial dans la formation de leur amour. Les deux jeunes amants découvrent l'amour à travers leur lecture commune du roman de Lancelot, et leur connaissance de l'amour évolue à mesure que leur lecture avance ${ }^{2}$. Ainsi, « La parole écrite exerce une véritable fascination. Elle pousse les deux adolescents à agir dans un sens déterminé ; elle est un miroir dans lequel ils se contemplent pour se découvrir semblables à leurs brillants modèles » (Girard, 2000 : 178).

10 C'est au cœur des histoires d'amour, où semble exalté "l'amour vrai ", que nous retrouvons donc les indices mimétiques les plus persistants. Selon Girard, cette donnée est clairement formulée dans les œuvres romanesques, Dante précédant ainsi Cervantès, Shakespeare ou Stendhal dans la dénonciation, derrière les illusions romantiques, du désir mimétique. Ce qui lui fait dire, résumant par la même occasion la médiation externe, que :

le génie de Dante, comme celui de Cervantès, est lié à l'abandon du préjugé individualiste. C'est donc l'essence même de ce génie qui est méconnu par le romantisme et ses séquelles contemporaines. Cervantès et Dante ouvrent, sur l'essence de la littérature, un domaine de réflexion qui inclut le "play within the play » shakespearien et la mise en abîme gidienne. (...) Le héros du désir dérivé cherche à conquérir l'être du modèle par une imitation aussi fidèle que possible. Si ce héros vivait dans le même univers que ce modèle, au lieu d'être à jamais séparé de lui par toute la distance du mythe ou de l'histoire, (...) il en viendrait forcément à désirer les mêmes objets que lui (Girard, 2011: 54).

11 Ce qui gêne Girard c'est que les œuvres concernées sont présentées comme propageant une idéologie qu'elles mettent en vérité à bas, et que la relation des amants littéraires avec leurs modèles romanesques antérieurs révèle plus de leur part un désir dérivé qu'un sentiment spontané et inconditionnel, malgré tous les efforts critiques à occulter cette donnée essentielle.

Une même censure intérieure efface toute perception du médiateur, supprime toute information du monde contraire à la "vision du monde " romantique et solipsiste. George Sand et Alfred de Musset partant pour l'Italie se prennent pour Paolo et Francesca mais jamais ils ne doutent de leur spontanéité. Le romantisme fait de la Divine comédie un nouveau roman de chevalerie (Girard, $2011: 52$ ).

12 L'œuvre de Shakespeare serait soumise aux mêmes interprétations (cf. Levin, 1986). Les adaptations du Songe d'une nuit d'été, dès le xix ${ }^{\mathrm{e}}$ siècle, sont ainsi dénaturées quand la pièce est revue à la lumière de l'idéalisme romantique de Coleridge, ou du «lyrisme préraphaélite » qui impose la doctrine d'une prééminence de l'amour-passion dans le corpus shakespearien (cf. Lewis, 1969).

Girard ne développe néanmoins pas sa proposition ex nihilo. Avant lui, La Rochefoucauld souligne dans ses maximes la médiation littéraire dans notre comportement amoureux, présentant l'amour non comme une découverte anthropologique mais comme une invention culturelle : il écrit qu' « il y a des gens qui n'auraient jamais été amoureux s'ils n'avaient jamais entendu parler de l'amour » (La Rochefoucauld, 1999 : 104).

14 Plus tard, au Xxe siècle, Denis de Rougemont développe pour sa part cette idée dans son examen historique et littéraire de l'amour-passion: "Si la littérature peut se vanter d'avoir agi sur les mœurs de l'Europe, c'est à coup sûr à notre mythe [de Tristan et Yseut] qu'elle le doit » (Rougemont, $2001: 190-191)$. 
15 Avant d'être une expérience de vie, l'amour-passion est une création orale puis écrite ; avant d'être une affaire du cœur, c'est une affaire de rhétorique. L'auteur souligne l'influence positive du mythe sur les mœurs du temps et sur l'évolution du sentiment amoureux. Il élabore cette thèse dans Les Mythes de l'amour. Tout en affirmant que les mythes, comme les lois, relèvent du générique, qu'ils nous conduisent au type là où la personne recherche un amour constamment neuf, il se pose néanmoins la question suivante : les mythes sont-ils notre création, ou nous les leurs? Il en conclut que "sans ces mythes, les Européens ne seraient pas ce qu'ils sont, n'aimeraient pas comme ils aiment, et leurs passions seraient incompréhensibles : car elles naissent de leurs rêves et non de leurs doctrines » (Rougemont, $1996: 28$ ).

Les mythes de l'amour déterminent notre conduite individuelle, des choix dont nous croyons être les seuls auteurs, au point que dans nos comportements amoureux nous laissons agir, au lieu de nous-mêmes, une image idéalisée et exemplaire de nousmêmes. Plus tard, Woody Allen se constitue comme témoin, dans son style propre, avec cette déclaration autobiographique dans un entretien avec le magazine Rolling Stone en septembre 1993 : « Je ne me suis jamais senti à l'aise dans le monde réel. Je crois que ma génération a grandi avec un système de valeurs fortement marqué par les films... Mes idées de l'amour (romance) venaient du cinéma ${ }^{3}$ ». Woody Allen fait d'ailleurs revivre la génération qu'il évoque ici dans The Purple Rose of Cairo (1985) et Radio Days (1987). Dans cette tendance du cinéma contemporain, le cinéma classique exerce une influence symbolique et devient vecteur de désir et médiateur externe pour les personnages d'Allen, comme le cinéma, en tant que médium, l'est pour nous.

\section{Pour une tentative " girardienne » de définition du comique}

17 À ce stade, nous devons nous demander si ces différentes considérations de notre rapport à la littérature, ne se résumant pas nécessairement au récit comique, ne révèlent pas par contre une donnée essentielle dans la syntaxe de la comédie. Girard le suggère lui-même en rapprochant comédie et tragédie à partir d'un schème fondamental qui rappelle explicitement la motivation de Don Quichotte aussi bien que celles de héros tragiques (d'CEdipe à Macbeth) : celui d'un " présomptueux victime de sa présomption" - dans le cas de la tragédie, Aristote parle de l'hamartia, l'erreur tragique. Mais Girard essaie désormais de comprendre de quelle manière ce schème structurel détermine spécifiquement la dynamique interne de la comédie, puisqu'il y produit un effet inverse à celui que produit une tragédie, ou un mélodrame moderne.

18 C'est bien plus tard que Girard va développer ses réflexions sur la comédie, en soulignant dans le fonctionnement du comique et dans les mécanismes du rire, une relation triangulaire centrale. Dans un texte publié en 1987 et repris en 2002 dans La Voix méconnue $d u$ réel, intitulé "Un équilibre périlleux: essai d'interprétation du comique ", Girard va enfin aborder de front le sujet. Ce texte n'est aucunement relié à ses études passées sur Shakespeare ou Cervantès - il ne cite d'ailleurs à aucun moment les deux auteurs. Il s'intéresse plutôt à la théorie du comique, en s'appuyant sur le texte d'Henri Bergson, Le Rire (1900), et propose une interprétation du comique, comme il le dit lui-même, qui pourrait justifier ses propres intuitions mimétiques. 
Le contexte de la rédaction de ce texte est bien différent de celui de ses écrits sur Shakespeare et Cervantès. La théorie du désir mimétique est désormais bien consolidée dans le champ des sciences humaines. Née certes dans l'analyse des textes littéraires, elle connait ensuite une légitimité croissante dans le domaine de l'anthropologie philosophique, discipline dans laquelle Girard va désormais s'engager lui-même. La démarche de l'auteur est donc différente. Il cherche non à prouver la validité de son système, mais à lui trouver des ramifications multiples, aussi bien en psychanalyse, en histoire, qu'en sociologie.

C'est dans ce sens que la comédie, en tant que genre narratif reconnaissable et reconnu, lui semble être un vecteur aussi légitime que d'autres pour la révélation de la vérité mimétique. Dans son texte, Girard commence par suggérer la fonction centrale du conflit, et plus particulièrement du conflit triangulaire, dans le fonctionnement du comique. Ses premières considérations rappellent les arguments de Bergson qui, au début du siècle, affirme déjà que « Le comique naîtra, semble-t-il, quand des hommes réunis en groupe dirigeront toute leur attention sur un d'entre eux, faisant taire leur sensibilité et exerçant leur seule intelligence » (Bergson, $2007: 6$ ). Il identifie surtout l'objet du rire comme un être dont la raideur morale et émotionnelle l'isole du reste de la communauté. Ainsi, « quiconque s'isole s'expose au ridicule, puisque le comique est fait, en grande partie, de cet isolement même» (Bergson, 2007 : 106). La fameuse formule de la «mécanique plaquée sur du vivant " souligne une opposition entre ce mouvement de fluidité et de grâce qu'est «l'élan vital», concept central de sa philosophie, et tout comportement raide et figé.

Bergson suggère ainsi dans le comique un processus d'exclusion où le sujet définit sa position de rieur en fonction de tiers stigmatisés ${ }^{4}$. Le rire naît ainsi quand on reconnait dans les autres un comportement qui n'est plus organique, humain, là où il est censé l'être. Nous rions ainsi de l'autre quand il commence à agir de manière qui le déshumanise. Nous retrouvons cette idée bergsonienne, en filigrane, dans le principe de la médiation externe : le sujet du désir, en modelant son comportement sur celui d'un médiateur, "mécanise », si l'on peut dire, un comportement et des actions qui sont censés être spontanés, dans la tradition «Don Quichottesque ». Dans un autre sens, plus un individu s'abandonne inconsciemment au mimétisme, et plus il se condamne à agir sans spontanéité, d'autant plus qu'il est convaincu (ou du moins essaie-t-il de se convaincre) de l'intégrité de son geste et de ses choix.

La définition de Bergson repose sur une dichotomie entre le centre, le social (les actions et dispositions de la vie individuelle et sociale, déterminées par la notion de fluidité) et la marge (raideur du corps, de l'esprit, de caractère). L'efficacité du rire suppose un bon positionnement du spectateur par rapport à l'action comique : le comique fonctionne quand il offre le spectacle d'une mécanique de la vie, spectacle perçu à partir du vivant. Dans cette opposition entre raideur et harmonie sociale, le rire prend l'aspect d'un rituel symbolique de mise à mort: "Cette raideur est le comique, et le rire en est le châtiment » (Bergson, $2007:$ 16) ${ }^{5}$. Charles Mauron relève la même idée chez Hobbes qui fait du rire l'expression d'un sentiment de triomphe : « La théorie du rire triomphal se reliait, dans la pensée de Hobbes, à l'idée plus générale d'une lutte universelle : Bellum omnium contra omnes. Elle supposait un vaincu » (Mauron, $1970: 145)^{6}$.

Girard commence son article en mettant encore plus l'accent que Bergson sur le rôle du sujet-rieur dans la «déshumanisation » de l'objet du rire, pour mieux expliciter la dynamique conflictuelle à la source du comique. Ainsi, le comique repose sur un conflit 
à trois termes, telle que Véronique Sternberg l'affirme: "anomalie perçue avec distance par le sujet riant, et sans conséquence aucune pour l'objet (victime) du rire ", le comique suppose une relation triangulaire entre un émetteur (l'artiste), un objet comique et un récepteur (Sternberg-Greiner, $2003: 17$ ).

Girard reprend certes l'idée de Bergson, mais il inverse dans un sens, sans vraiment le souligner, le point de vue, puisque c'est l'objet du rire en tant que victime expiatoire qui l'intéresse. Ce n'est plus le groupe qui dirige son attention vers un individu exclu, mais c'est celui qui fait rire qui essaie, vainement, de nier toute forme de réciprocité entre lui et les autres, le rire, « en tant qu'affirmation d'une supériorité, [constituant ainsi] une négation de la réciprocité » (Sternberg-Greiner, $2003: 19$ ).

Cette caractéristique du comique identifiée par Bergson et reprise par Girard est donc double, puisqu'elle met en lumière un processus d'inclusion-exclusion au cœur du dispositif comique, mais aussi et surtout l'incapacité du protagoniste comique à se conformer à l'image qu'il se fait de lui-même, passant ainsi du statut de sujet à d'objet risible. Girard suggère que "les schèmes structurels du comique contestent la souveraineté [de l'individu, cela s'entend] plus radicalement que la divinité ou la destinée [comme dans la tragédie] (Girard, 2004: 272). Il ajoute plus loin "qu'un individu tente d'imposer à ce qui l'entoure ce qu'il croit être sa propre règle individuelle. On se met à rire quand, très soudainement et de façon spectaculaire, cette prétention vole en éclats » (Girard, $2004: 274$ ).

Girard nous ramène, sans le nommer, à ce qui constituait la nature fondamentale de Don Quichotte, et à l'articulation principale de la médiation externe du désir. Ce qui avait servi à expliciter les mécanismes de sa théorie mimétique est réinvesti, dès son article de 1987, pour définir une donnée syntaxique possible de la comédie et de l'esthétique comique.

Girard s'attaque ici à un problème ardu, mais passionnant: le genre comique, et le comique en tant que mode de communication, est un sujet particulièrement périlleux à chaque fois qu'on le soumet à un exercice de théorisation. Si le texte de Bergson, qui traite plus du comique que du rire (contrairement à ce que suggère le titre) a depuis acquis une légitimité canonique, il n'en demeure pas moins que, dans les études génériques, la question de la comédie demeure fuyante et problématique à partir du moment où on cherche à lui attribuer une sémantique propre - des codes narratifs, visuels, des thématiques, etc.

Mais l'inclusion de ce texte dans un ouvrage comme $L a$ voix méconnue du réel s'est faite pour cette raison même : réfléchir sur ce qui, aux yeux de la méthode scientifique, semble indéfinissable (à la lecture des autres textes de l'ouvrage, nous avons en effet l'impression que c'est le seul point sur lequel le texte sur le comique rejoint les autres). Le comique semble un défi pour Girard, et, avec ses analyses sur des textes comiques, il cherche à formuler, des années plus tard et le plus clairement possible, une synthèse sur l'essence du comique. Et bien qu'il ne renvoie, à aucun moment, au roman de Cervantès (pour un texte sur le comique, les renvois à des œuvres du genre sont étrangement rares), l'intuition principale de Girard aurait été d'identifier en Don Quichotte, de manière peut-être accidentelle mais heureuse, le prototype du héros comique moderne. 


\section{Don Quichotte, premier héros comique moderne?} modernes, littéraires, théâtrales ou cinématographiques, est en effet cette incapacité du personnage à rendre ses actes conformes à l'image qu'il se projette de lui-même. Chez les héros du Vaudeville français, des opérettes de la « Mitteleuropa » massivement adaptées au cinéma dès les années 1930 (cf. Por, 2011), ou de la comédie de mœurs anglaise, il existe ce que Francis Farrugia appelle une "dissonance cognitive et émotionnelle» (Farrugia, 2010:5) entre eux et leur temps. Cette dissonance est le résultat d'une " consonance narrative » entre ces héros comiques et l'univers qu'ils se sont constituées, à partir de leurs lectures ou de leurs expériences de spectateurs nous voyons apparaître en effet, au théâtre puis au cinéma, de plus en plus de situations dramatiques où les personnages sont eux-mêmes dans une position de spectateurs passifs et subjugués en face d'un spectacle dans le spectacle. Leur conscience entre en conflit avec leur expérience, puisqu'elle introduit dans la réalité diégétique une scission entre réalité effective et univers fictif. Le personnage se constitue un double de lui-même, l'un portant vers le bas (c'est-à-dire le corps, le familier), l'autre vers l'ailleurs, tourné vers les affaires complexes de l'esprit (c'est-àdire l'étrange, le fascinant). manquent pas d'exploiter. Elle permet de définir les relations des personnages entre eux, et leurs fonctions interchangeables de sujets du rire (ceux qui rient avec le spectateur) et objets du rire (ceux vers qui notre rire est dirigé).

Connées ne sont pas exclusives à la comédie. Un des grands problèmes dans la définition de la comédie en tant que genre est justement de lui trouver une sémantique et une syntaxe propres - puisque la spécificité du genre comique est d'emprunter les conventions narratives et visuelles d'autres genres qui lui sont contemporains pour les détourner. Ainsi, la comédie fonctionne moins sur l'identification par le spectateur d'un contenu que sur la fonction «perlocutoire » du genre, c'est-à-dire l'expérience d'un effet (en l'occurrence le rire). Mais ces données révèlent par contre quelques-uns de ses rouages esthétiques et narratifs essentiels. Nous pouvons dans ce cas en introduire trois : la présence dans la diégèse d'un sujet-rieur, double du spectateur ; la fusion des types comiques traditionnels de l'eiron et de l'alazon dès le $\mathrm{XVII}^{\mathrm{e}}$ siècle; la catharsis comique.

Premièrement, nous voyons dans ce cas une convention comique s'imposer de plus en plus, dans les comédies théâtrales et cinématographiques : la présence d'un spectateurrieur dans le spectacle qui se déroule devant nous. Ce personnage-spectateur agit comme un miroir de nous-mêmes, médiateur de notre rire, son rire amplifiant l'expérience du spectateur. Le rire a ainsi un caractère mimétique que Bergson a déjà suggéré : «On ne goûterait pas le comique si l'on se sentait isolé. Il semble que le rire ait besoin d'un écho» (Bergson, $2007: 4$ ). Cette illustration diégétique du rire du 
spectateur, nourrie d'une "verve satirique " indéniable, ainsi que d'un "austère pessimisme » selon les mots du philosophe, s'exerce aux dépens d'un personnage qui apparaît dans toute sa faillibilité humaine. Le personnage-rieur dirige ainsi notre attention vers l'objet du rire, s'alliant à nous contre ce dernier, nous permettant ainsi d'identifier plus clairement la vanité, la présomption, les multiples prétentions de l'objet $\mathrm{du}$ rire, puisque le rire a besoin d'une situation claire pour pleinement s'exprimer. Girard devine cela en affirmant que

Notre maîtrise et notre autonomie croissent à mesure que nous voyons les autres perdre les leurs et le piège se refermer sur eux. (...) N'importe quel clown ou comédien au fait de son métier sait parfaitement que les gens riront à ses propres dépens ou aux dépens d'une tierce personne (Girard, 2004 : 282-283).

Dans les comédies modernes, cela se traduit par la convention comique du triangle amoureux, topo dramatique largement exploité pour des raisons plutôt évidentes (son potentiel commercial et les combinaisons narratives qu'il promet), mais qui participe surtout de manière organique aux mécanismes du rire. La réinvention du couple comique, puisque ses mésaventures comiques consistent à le mettre à l'épreuve et à le faire sortir triomphant, malgré tout, de toutes les tentations imaginables, se réalise à partir de la capacité des membres du couple à vivre, mais aussi à rire ensemble, et ce rire se fait généralement aux dépens d'un tiers. Or les places étant permutables, le protagoniste n'est pas immunisé contre le ridicule: il peut aussi bien subir un rituel d'humiliation qu'en être l'instigateur. La comédie, dès le XvII siècle, avec Shakespeare en Angleterre (Beaucoup de bruit pour rien), Lope de Vega en Espagne (Le Chien du jardinier) ou Corneille ( $L a$ Galerie du palais, La Place royale) et Molière en France, expérimente avec la typologie des personnages, modifiant leur nature et leur réception par le public, les rendant plus vulnérables, plus perméables au ridicule, ce qui nous renvoie au point suivant.

Pour le deuxième point de rupture dans les modes comiques, Girard a eu l'intuition d'un grand changement dans la typologie des personnages comiques. Initié par Shakespeare mais rendu systématique depuis le xix ${ }^{\mathrm{e}}$ siècle, ce changement concerne la dynamique entre l'eiron, le personnage qui se prend pour moins qu'il n'est, et l'alazon, le personnage qui se prend pour plus qu'il n'est, généralement désigné par le prétentieux ridicule. La comédie repose habituellement sur la distinction nette de ces deux rôlestypes du démystificateur et de l'imposteur. Mais, séparés d'abord, ces deux types comiques se confondent de plus en plus dès le XVII ${ }^{e}$ siècle, pour offrir un héros comique aussi sympathique que vulnérable à la dérision. Cette tendance devient plus claire dans le théâtre comique du XIX ${ }^{e}$ siècle, aussi bien dans le vaudeville français, la comédie de mœurs anglaise ou les farces des pays de la «Mitteleuropa ». N. T. Binh et Christian Viviani ne manquent de souligner la présence de cette figure, de «jeune premier drôle » dans le cinéma d'Ernst Lubitsch, cinéaste allemand émigré aux Etats-Unis, et dont l'œuvre forme une sorte d'extension naturelle du théâtre européen dont il a régulièrement adapté à l'écran les œuvres les plus populaires (The Merry Widow, The Love Parade, Design For Living). Il brise ainsi cette " convention [qui] voudrait que le bouffon soit nécessairement le personnage secondaire, le faire-valoir du héros romantique. Dans une comédie, le premier fait rire à ses dépens (ridiculus), le second en maniant consciemment l'humour (ridendus)» (Binh et Viviani, 1991: 56). Ces deux compétences comique et romantique, du bouffon et du jeune premier, fusionnent désormais dans le même personnage, ce qui aide à souligner un fléchissement de la comédie romantique vers les modes de la satire ou de l'ironie. 
période où Girard dénonce le «mensonge romantique » (dès 1961), parle également de l'illusion romantique mise à nu et ridiculisée par le jeune Gustave Flaubert dans sa correspondance.

Leur [les Blasés] rire les venge d'abord des romantiques, ces imposteurs: c'est Werther et non pas Goethe qui s'est fait sauter la cervelle; c'est Chatterton et non Vigny qui s'est empoisonné. Les messieurs de Paris voudraient faire croire qu'on meurt d'amour et de spleen, c'est faux: les grands sentiments, ils en vivent ou plutôt ils vivent d'en écrire car ces mortelles passions n'existent pas (Sartre, 1971: 1441).

Mais contrairement à Girard, qui ne le suggérera que bien plus tard, Sartre associe déjà, de manière intuitive, notre relation complexe aux modèles romantiques au processus même du comique, et la capacité du comique à dénoncer, à travers ces illusions, la disposition de l'homme à se complaire dans une image faussée de lui-même et du monde. Le philosophe parle du comique en y soulignant le rôle du hasard dans la représentation comique de la relation de l'homme au monde :

Le hasard (...) apparaît, dans les constructions comiques, comme le principe négatif par excellence : c'est lui qui porte sentence et décrète que l'homme est impossible. Ce n'est pas un hasard si tout y arrive par hasard. La personne humaine s'y affirme d'abord comme souveraine, avec la conviction d'agir sur le monde et de gouverner sa vie. Le hasard vient ensuite, dénonçant cette illusion : le monde est allergique à 
l'homme, le comique nous rend témoins d'un processus de rejet. (...) [L'homme] sort déshumanisé de l'aventure puisque ses fins lui ont été volées et restituées au dernier moment par les choses (Sartre, 1971 : 1438-1439). véritable, le comique fait sentir son inéluctabilité. Mais surtout, à travers cette donnée, il fait ressortir la vanité de l'homme et le décalage entre l'image que nous nous donnons de nous-mêmes et de notre être. Ce qui pousse Sartre à faire ce constat sur le fond ontologique du comique : «Le rire spontané dénonce que cet individu - qui se prend au sérieux - n'est qu'un sous-homme. Le rire provoqué (par le comique) prétend nous révéler que tout homme est un sous-homme qui se prend au sérieux " (Sartre, $1971: 1440)$.

Même si Sartre ne parle pas dans ces quelques pages de Don Quichotte, nous reconnaissons dans les mésaventures de ce dernier une crise du sujet qui est devenue, dans notre modernité, et avec des penseurs comme Nietzsche ou Freud, constitutive de notre rapport au monde : notre volonté d'expliquer un monde que nous ne comprenons pas, et de maîtriser notre nature humaine sur laquelle nous n'avons qu'une prise partielle.

Pour conclure, j'ai tenté de relier les analyses proposées par Girard de Don Quichotte avec ses considérations, bien ultérieures, sur le comique, de manière à rendre compte de la dimension parodique et de la syntaxe de la comédie, et de relever chez les héros comiques au théâtre et au cinéma des traits qui en font les descendants du prototype «quichottien». Ainsi, j'essaie d'identifier chez les héros comiques modernes, du théâtre (par exemple le vaudeville français et " mitteleuropéen ", la comédie de mœurs anglaise - Maugham, Wilde, Shaw) et du cinéma (par exemple la comédie américaine classique) un "syndrome de Don Quichotte ", qui les rend victimes d'une appropriation mimétique de modèles illusoires, leur attachement à des expressions conventionnelles du désir les entraînant dans une série de mésaventures comiques avant un retour, pénible et inévitable, à la lucidité.

\section{BIBLIOGRAPHIE}

BERgSon, Henri (2007). Le Rire. Paris : Presses Universitaires de France.

BINH, n.t., Viviani, Christian (1991). Lubitsch. Paris : Rivages/Cinéma.

DANTE (1956). La Divine comédie. Paris : Garnier.

EL-KHOURY, Toufic (2016). La comédie hollywoodienne classique (1929-1945). Structure triadique et médiations du désir. Paris : L'Harmattan, « Champs visuels ».

FARRUGIA, Francis (2010). «Le syndrome narratif : une 'inquiétante étrangeté’ », Sociologies [En ligne], Dossiers, Émotions et sentiments, réalité et fiction [disponible le1/6/2010] URL : http:/ sociologies.revues.org/index3152.html 
GIRARD, René (2004). «Un équilibre périlleux : essai d'interprétation du comique », in La Voix méconnue du réel, une théorie des mythes archaïques et modernes. Paris : Livre de poche, « Biblio essais ».

GIRARD, René (2000). Critiques dans un souterrain. Paris : Le Livre de Poche, « Biblio essais ».

GIRARD, René (2011). Géométries du désir. Paris : L'Herne.

GIRARD, René (1999). Mensonge romantique et vérité romanesque (1961). Paris : Hachette, « Pluriel ». LA ROCHEFOUCAULD, François de (1999). Maximes, réflexions, lettres. Paris : Hachette, « Pluriel ». MAURON, Charles (1970). Psychocritique du genre comique. Paris : José Corti.

LEVIN, Harry (1986). «Critical Approaches to Shakespeare from 1660 to 1904 », in Stanley Wells (éd.). The Cambridge Companion to Shakespeare Studies. Cambridge : Cambridge University Press.

LEWIS, Allan (1969). « Midsummer's Night Dream, Fairy Fantasy or Erotic Nightmare? », Educational Theater Journal, 21:3, pp. 251-258.

POR, Katalin (2011). De Budapest à Hollywood, le théâtre hongrois et le cinéma hollywoodien (1930-1943). Rennes : Presses Universitaires de Rennes, « Le Spectaculaire ».

RougEMONT, Denis de (2001). L'Amour et l'Occident (1939). Paris : Plon, « Bibliothèques 10/18 ». RougEmont, Denis de (1996). Les Mythes de l'amour (1972). Paris : Albin Michel, « Espaces libres ». SARTRE, Jean-Paul (1971). L'idiot de la famille, Gustave Flaubert de 1821 à 1857, Vol. 2. Paris : Gallimard, «NRF ».

scoufos, Alice-Lyle (1981). " The 'Paradiso Terrestre' and The Testing of Love in As You Like It », Shakespearean Studies, 14, pp. 215-227.

SHUMWAY, David (2003). Modern Love, Romance, Intimacy and the Marriage Crisis. New York : New York University Press.

STERNBERG-GREINER, Véronique (2003). Le Rire. Paris : GF-Flammarion, « Corpus », pp. 17-22.

\section{NOTES}

1. Il définit ainsi le terme qu'il introduit : «L'expression syndrome narratif désigne un ensemble de symptômes constitués d'un complexus de sentiments, représentations. Cet ensemble est caractéristique d'une certaine disposition émotionnelle qui s'étaye sur un lien étroit unissant durablement un ou des acteurs à des narrations sociales au sens large : à des discours, films, romans, avec lesquels ils entretiennent des relations identificatoires privilégiées et constructrices de leurs dispositions mentales et corporelles, et qui finissent par les constituer en un type relativement organisé autour d'une dynamique de la mimêsis inconsciente les disposant existentiellement sur le registre de la reproduction symbolique et de l'interprétation des symptômes élus » (Farrugia, $2010: 5$ ).

2. Francesca de Rimini s'adresse ainsi au poète qui lui demande comment elle et son amant sont tombés amoureux : "De savoir la racine première / De notre amour, si tu en as envie / Je serai celle-là qui pleure, mais qui dit / Certain jour, par plaisir, nous lisions dans le livre / De Lancelot comment amour le prit: / Nous étions seuls, sans nous douter de rien / A plusieurs fois cette lecture fit / Que, relevant les yeux, ensemble nous pâlimes. / Mais un passage seul a triomphé de nous : / Lorsque nous eûmes lu, du désiré sourire, / Qu'il fut baisé par un si bel amant / Lui, qui 
jamais de moi ne sera retranché, / Il me baisa, tout en tremblant, la bouche. / Le livre, et son auteur, fut notre Galehaut » (Dante, 1956 : 34-35).

3. "The real world is a place that I've never felt comfortable in. I think that my generation grew up with a value system heavily marked by films... My ideas of romance came from the movies " (Shumway, 2003 : 81).

4. «Toute raideur de caractère, de l'esprit et même du corps, sera donc suspecte à la société, parce qu'elle est le signe possible d'une activité qui s'endort et aussi d'une activité qui s'isole, qui tend à s'écarter d'un centre commun autour duquel la société gravite, d'une excentricité enfin [...]. Le rire doit être quelque chose de ce genre, une espèce de geste social » (Bergson, 2007 : 15).

5. Dans ses notes critiques, Fréderic Worms insiste sur cette caractéristique du rire chez Bergson : « Le rire apparaît comme un geste social répressif qui ne prend sens qu'en rapport à un 'signe', signe d'une tendance possible à l'isolement ou "symptôme » d'une insociabilité 'latente' [...]. Châtiment symbolique qu'est, en son essence, le rire spécifiquement provoqué par le comique » (Bergson, 2007 : 185).

6. Mauron cite On Human Nature (1650) du philosophe anglais : "Le rire n'est rien d'autre que la gloire soudaine émanant de notre prise de conscience d'une certaine distinction en nous, en comparaison avec l'infirmité des autres ou de nos tares antérieures » - «Laughter is nothing but the sudden glory arising from sudden conception of some eminence in ourselves; by comparison with the infirmity of others, or with our own formerly" (Mauron, 1970 :144). Cette idée est ensuite critiquée par Mauron.

\section{RÉSUMÉS}

Parodie des romans de chevalerie, Don Quichotte est construit sur l'incapacité du protagoniste à aligner ses désirs et ses actions sur ceux de ses héros fictifs. A partir du roman, René Girard développe la théorie de la médiation externe du désir, centrée sur la disposition d'un personnage à imiter et à se conformer aux actes d'un modèle qui n'appartient pas à son propre univers diégétique. Cette donnée, mise en lumière par Girard, n'est pas assez mise en avant dans le cadre d'études sur l'esthétique et les mécanismes du comique. Dans ce sens, nous identifions chez les héros comiques modernes ce que nous désignons par «syndrome de Don Quichotte", qui les rend victimes d'une appropriation mimétique de modèles illusoires, les plongeant dans une série de mésaventures avant un retour pénible et inévitable à la lucidité. Nous essayons ainsi de relever la spécificité du roman de Cervantès, sa manière d'expliciter la dimension parodique et la syntaxe de la comédie, et de relever chez les héros comiques modernes des traits qui en font les descendants du prototype « quichottien ».

Cervantes' Don Quixote, a parody of chivalry romances, centers on the misadventures of a protagonist who tries to align his desires and actions with those of his literary models. From this idea, René Girard develops his theory of external mediation of desire: the disposition of a character to imitate a model who does not belong to his diegetic universe. This idea, introduced by Girard, is not discussed enough in the theory and the aesthetics of comedy. Therefore, we identify in the modern comic heroes what we designate by a "syndrome of Don Quixote", that make them victims of a mimetic appropriation of illusory models, and plunge them in a series of misadventures before a painful and inevitable return to lucidity. We will try to highlight Don Quixote's specificity, its impact on parody and comedy's syntax, and identify what make modern 
comic heroes the descendants of Cervantes's prototype: incapable of desiring without models, and attached to conventional expressions of desire that lead them to disaster.

31 janvier 2018

INDEX

Keywords : Don Quichotte, Girard (René), Comedy, parody, Literature, Cinema, mimetic appropriation, mimetic desire

Mots-clés : Don Quichotte, Girard (René), Comédie, parodie, Littérature, Cinéma, appropriation mimétique, médiation du désir

\section{AUTEUR}

TOUFIC EL-KHOURY

Université Saint-Joseph de Beyrouth

toufic.khoury[at]usj.edu.lb 